\title{
Elevated Impulsivity and Impaired Decision-Making in Abstinent Ecstasy (MDMA) Users Compared to Polydrug and Drug-Naïve Controls
}

\author{
Michael John Morgan*,', Lara Chiara Impallomeni', Alessandro Pirona' and Robert David Rogers ${ }^{2}$ \\ 'Department of Psychology, School of Life Sciences, University of Sussex, Sussex, UK; ' Department of Psychiatry, Oxford University, Oxford, UK
}

\begin{abstract}
Ecstasy (MDMA; 3,4-methylenedioxymethamphetamine) has a well-recognized neurotoxic effect on central serotonergic (5-HT) systems in animals, and there is some evidence of persistent serotonergic dysregulation in human ecstasy users. Serotonin is believed to mediate impulsive behavior and effective decision-making. Thus, the aim of the present study was to investigate impulsive behavior and decisionmaking in abstinent regular ecstasy users. Three groups were compared: 'ecstasy users' (recreational ecstasy users who reported modest use of illicit drugs other than cannabis), 'polydrug controls' (ecstasy naïve illicit drug users), and 'drug-naïve controls'. All participants completed personal details and general drug history questionnaires, the National Adult Reading Test, Matching Familiar Figures Test (MFF20), a risky decision-making task (RDMT), and the Card Arranging Reward Responsivity Objective Test (CARROT). The groups did not differ on the CARROT measure of responsiveness to financial incentive; however, the ecstasy group displayed significantly elevated MFF20 impulsivity, and showed reduced discrimination between magnitudes of prospective gains and losses when making risky decisions, compared to the 'polydrug' and 'drug-naïve' control groups. These findings may reflect a vulnerability of 5-HT systems in the orbital prefrontal cortex and interconnected corticolimbic circuitry to the cumulative neurotoxic effects of ecstasy and have clinical significance for regular ecstasy users. The combination of elevated impulsivity and impaired use of reinforcement cues in uncertain decision-making may comprise risk factors for continued drug abuse and everyday functioning.

Neuropsychopharmacology (2006) 31, I 562-1573. doi:I0. I038/sj.npp. I 300953; published online 9 November 2005
\end{abstract}

Keywords: ecstasy; MDMA; 3,4-methylenedioxymethamphetamine; cannabis; decision-making; emotion; impulsivity

\section{INTRODUCTION}

Recreational use of 'ecstasy' (3,4-methylenedioxymethamphetamine, MDMA) at nightclubs, dance parties, and 'raves' has become increasingly widespread (eg Tasker et al, 1999; Johnston et al, 2000). The popularity of this drug has given rise to concern, since preclinical research has demonstrated that repeated doses of MDMA can cause serotonergic neurodegeneration in animals (eg Ricaurte et al, 1992, 2000; Steele et al, 1994; Green et al, 1995), and there is some evidence that chronic consumption of ecstasy is associated with protracted dysregulation of 5-HT systems in humans. Drug-free ecstasy users have been found to have low levels of 5-HT, and its metabolite 5-HIAA (McCann et al, 1994; Kish et al, 2000). Additionally, neuroimaging studies suggest that extensive exposure to ecstasy may deplete

*Correspondence: Dr MJ Morgan, Department of Psychology, School of Life Sciences, University of Sussex, Falmer, Brighton, BNI 9QG, UK, Tel: 01273 877202, Fax: 01273 6786 II,

E-mail: m.j.morgan@sussex.ac.uk

Received 22 April 2005; revised 21 September 2005; accepted 23 September 2005

Online publication: 29 September 2005 at http://www.acnp.org/ citations/Npp092905050265/default.pdf
5-HT in humans (eg McCann et al, 1998; Semple et al, 1999; Reneman et al, 2000), although such effects appear to recover after prolonged abstinence (Reneman et al, 2001; Thomasius et al, 2003; Buchert et al, 2004).

There is also growing evidence that ecstasy use is associated with particular neuropsychological impairments. Compared to ecstasy-naive illicit drug users, recreational ecstasy users have been reported to exhibit persistent impairments of episodic memory and 'executive functions', including deficits in attention and working memory (eg Morgan, 1999; McCann et al, 1999; Gouzoulis-Mayfrank et al, 2000, 2003; Rodgers, 2000; Wareing et al, 2000, 2004; Verkes et al, 2001; Fox et al, 2002; Morgan et al, 2002; Hanson and Luciana, 2004; von Geusau et al, 2004). Impaired 'executive functions' and 5-HT depletion are both thought to play a role in impulse control (eg Evenden, 1999). On the basis of pre-clinical and clinical data, Soubrié (1986) proposed that a reduction in serotonergic activity facilitates active responses at the expense of behavioral inhibition and can result in impulsive behavior in circumstances where an active response is inappropriate and results in either an aversive event or omission of reinforcement. This is in line with clinical evidence that low levels of 5-HIAA are associated with impulsive behavior in 
the context of certain psychiatric disorders (eg Coccaro, 1989; Linnoila et al, 1993) and with evidence that neuroendocrine responses to fenfluramine are significantly elevated in substance abusers with higher levels of impulsivity (Fishbein et al, 1989).

In support of the hypothesis that heavy ecstasy use may result in cognitive impulsivity, we reported that regular ecstasy users commit more errors on a paper and pencil version of the 20-item Matching Familiar Figures Test (MFF20; Cairns and Cammock, 1978) - a measure of 'reflection impulsivity' than either matched polydrug control participants or nondrug controls (Morgan, 1998). Subsequently, we replicated this finding in a study of current and ex-ecstasy users. Both groups of ecstasy users exhibited elevated MFF20 impulsivity compared to ecstasynaïve polydrug users and drug-naïve controls (Morgan et al, 2002). By contrast, investigations of other behavioral measures of impulsivity have yielded negative results. Gouzoulis-Mayfrank et al (2003) reported that there was no difference in the performance of a Go/No Go task between nonusers, moderate, and heavy ecstasy users despite the evidence that the ecstasy users exhibited poorer memory performance.

The lack of consensus in the latter literature may reflect differences in the neuropharmacology of various subtypes of impulsivity (eg Winstanley et al, 2004). In agreement with our MFF20 findings in ecstasy users, Evenden (1999) reported that administration of a $5-\mathrm{HT}_{2}$ antagonist elevated impulsivity in an animal model of 'reflection-impulsivity' performance, but did not affect other animal models of impulsivity including the inhibitory control subtype assessed by the Go/NoGo task. Furthermore, administration of a $5-\mathrm{HT}_{2}$ agonist reduced 'reflection impulsivity', but elevated impulsivity in animal models of inhibitory control and temporal discounting.

Recently, we have developed a computerized version of the MFF20 to provide a convenient and easily administered measure of reflection impulsivity. Specifically, in the present study, we utilized this variant of the test in samples of ecstasy users, ecstasy-naïve polydrug users, and drugnaïve controls to test the reliability of our earlier findings and to initiate a systematic analysis of the association between ecstasy use and impulsive performance in measures of other kinds of choice behavior.

Another manifestation of impulsivity is in the context of decision-making. Real-life decision-making, involving choices between actions leading to uncertain rewards and penalties, depends on the effective processing of information about the probability and value of different outcomes, to arrive at adaptive choices. The cognitive functions underpinning this activity will include the ability to attend to such stimuli, integrate information about the value of different outcomes (using a variety of normative and nonnormative heuristics; Kahneman and Tversky, 1979), and select appropriate responses while inhibiting inappropriate options. Therefore, impaired decision-making may reflect a variety of impulse control problems.

Research with neurological patients and brain-imaging technologies has demonstrated that the capacity to make effective real-life decisions depends upon the integrity of the orbitofrontal cortex and its neurochemical innervation (Bechara et al, 1996; Rogers et al, 1999a, b, 2004b; Scarna et al, 2005). Recent data suggest that chronic substance abusers show marked deficits in decision-making using instruments known to be sensitive to focal lesions of orbital prefrontal cortex (PFC) (Bechara et al, 2001; Grant et al, 2000; Rogers et al, 1999a). Other data have suggested that at least some of these deficits can be mimicked by acute depletion of L-tryptophan (Rogers et al, 1999a, b), consistent with reports of depleted 5-HT in the orbital regions of PFC of methamphetamine abusers (Wilson et al, 1996).

In the present study, we investigated the decision-making of ecstasy users, ecstasy-naïve polydrug users, and drugnaïve controls using a recently devised paradigm, which measures decision-makers' attention to reinforcement signals involved in risky choice: the magnitude of prospective gains (or reward), the magnitude of prospective losses (or punishment), and the probabilities with which each of these outcomes will be delivered (Rogers et al, 2003, 2004a,b; Scarna et al, 2005). Previously, tryptophandepleted participants showed reduced discrimination between magnitudes of prospective gains associated with different choices in this task (Rogers et al, 2003). There is some evidence that substance dependence can compromise more general incentive-motivational processing (eg Harris and Aston-Jones, 2003). To determine if cognitive deficits were confounded by reduced motivation, a behavioral measure of responsiveness to financial incentive - a computerized version of the Card Arranging Reward Responsivity Objective Test (CARROT; Powell et al, 1996) in which participants sort stimuli under conditions of nonreward and reward - was employed in the present study. To control for any group differences in premorbid intelligence, we employed the National Adult Reading Test (NART; Nelson, 1982). Although we did not seek to test ex-ecstasy users in the present study, the three other groups of participants were similar to those in our previous study (Morgan et al, 2002). However, in contrast to our previous studies, in the present study we succeeded in recruiting primarily current ecstasy and/or cannabis-only users, thereby reducing confounding by histories of other illicit drug use.

\section{METHOD}

The study was approved by the University of Sussex Ethics Committee. All participants provided written informed consent.

\section{PARTICIPANTS}

In all 59 young adults (aged 20-26 years) were recruited through personal contacts and by word of mouth. All participants were students in higher education, attending various English Universities, and were selected on the basis of a confidential screening interview about their illicit drug use into one of three groups comprising 19-20 participants each:

'Ecstasy' Group (11 males and nine females): Inclusion required good health, minimum consumption of 50 ecstasy tablets, abstinence from ecstasy for at least 5 days, and abstinence from cannabis for $12 \mathrm{~h}$ prior to testing. Participants were recruited who primarily used ecstasy and cannabis and reported only modest use of other illicit 
drugs (as indicated by the screening interview and General Drug Use Questionnaire).

'Polydrug' Group (12 males and eight females): Inclusion required participants to meet the above criteria and have a similar history of consumption of cannabis and other illicit drugs to the ecstasy group, but have no history of exposure to ecstasy.

'Drug-nä̈ve' Group (nine males and 10 females): The same inclusion criteria applied; however, allocation to this control group required a history of no illicit drug use.

Exclusionary criteria for all groups included English as a second language, prescribed medications and serious health problems which could interfere with the nature of the study, previous substance dependence, a self-reported history of severe mental illness or psychosis, use of intoxicating doses of cannabis or alcohol within $12 \mathrm{~h}$ of assessment, or consumption of ecstasy within 5 days of assessment. Participants were administered the NART (Nelson, 1982) to provide an estimate of verbal IQ and ensure adequate knowledge of English (subjects had to obtain more than 25 correct answers to participate). Of the 59 participants tested, all 32 males and 27 females satisfied the inclusion criteria. The mean age for males was $22.03( \pm 1.44)$ years and for females was $22.09( \pm 1.27)$ years.

\section{MATERIALS}

Materials comprised two questionnaires and the NART test (Nelson, 1982). A Sony Minidisc and Hitachi microphone were used to record the participants while completing the NART test. The three computerized neuropsychological tests were administered on a DELL 'Inspiron 2500' laptop computer, with 12 by 9 inch screen.

\section{Questionnaire Measures}

(1) Personal Details Questionnaire: A 19-item demographic questionnaire was employed to ascertain personal details, including gender, age, education attained, medical history, psychiatric history, smoking status and time since last meal, and use of alcohol, cigarettes, and cannabis.

(2) General Drug Use: Participants completed a structured questionnaire (Morgan, 1998, 1999, 2002) which required them to indicate the age at first use, total duration, frequency, time since last use, and average dose of each substance ever consumed from an exhaustive list of illicit and prescribed substances.

(3) NART (Nelson, 1982): This vocabulary test provides an estimate of premorbid full IQ and ensured adequate knowledge of English. Participants were recorded reading a list of 50 grammatically irregular words (unusual in respect to the common rules of pronunciation).

\section{Neuropsychological Measures}

(1) Matching Familiar Figures Test (MFF20): Participants were administered a computerized edition of the 20item MFF20 test. The MFF20 was derived from the original Matching Familiar Figures Test (Kagan et al,
1964) by Cairns and Cammock (1978) and serves as a behavioral measure of impulsivity. The test format of the computerized MFF20, involves simultaneous presentation of a target figure centered on the left half of the screen and an array of six similar alternatives, all, except one, of which differ in one or more details presented in two columns on the right-hand side of the screen, with their corresponding numbers shown below each figure. Participants are required to select the figure that matches the target exactly, by pressing the number corresponding to the figure on the computer keyboard. Errors are signalled by a beep and when this happens participants are required to give another answer. Each participant is given 20 test items. Two measures were automatically recorded: the mean latency to first response and the total number of errors committed. A third composite index of impulsivity, the 'I' score (Salkind and Wright, 1977; Messer and Brodzinsky, 1981), was calculated by subtracting the standard score of the mean latency to first response from the standard score of the total number of errors committed $\left(Z_{\mathrm{e}}-Z_{1}\right)$.

(2) CARROT: Participants were given a computerized version of the Powell et al (1996) CARROT task to provide a behavioral measure of the behavioral responsiveness to small financial incentive. Participants were required to sort computerized cards into categories containing a 1,2 , or 3 as quickly as possible. Time taken in the first baseline trial (T1) provided the individualized time limit for the subsequent three trials (T2, T3, and T4). In T3, the rewarded condition (REW), participants received (and retained) 10 pence for every five presentations sorted correctly. T2 and T4 (unrewarded) were averaged to provide the nonreward (NONREW) index. Rate of sorting was recorded automatically for each individual trial. A reward responsiveness index was derived by subtracting the NONREW rate from the REW rate.

(3) Risky decision-making: Participants were asked to make a series of choices between binary-outcome gambles. Each gamble was represented visually by a histogram, the height of which indicated the relative probability of gaining a given number of points. The possible gains were indicated in green ink above the histogram; possible losses were indicated in red ink underneath the histogram. On each trial, one gamble (colored yellow) was the control gamble, consisting of a 0.50 probability of winning 10 points and a 0.50 probability of losing 10 points. The alternative 'experimental' gamble (colored blue) varied in the probability of winning which was either high or low (0.66 vs 0.33 ), possible gains which were large or small (80 vs 20 points), and possible losses which were either large or small (80 vs 20 points). These variables were combined, in a crossed design, to produce eight trial types. Figure 1 shows an 'experimental' gamble with a 0.33 chance of winning 80 points (and a 0.66 chance of losing 20 points).

The control and 'experimental' gambles gamble appeared randomly on the left and right of the display. The volunteer was required to press the ' 1 ' or ' 2 ' key on the computer 


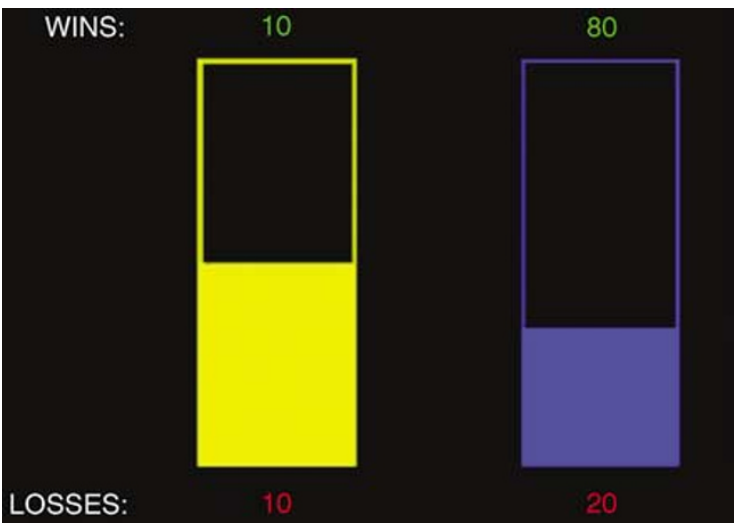

Figure I One example trial from the decision-making paradigm consisting of an 'experimental' gamble with a 0.33 chance of winning 80 points and a 0.66 chance of losing 20 points vs the control gamble with a 0.50 chance of winning 10 points and a 0.50 of losing 10 points.

keyboard to indicate choice of the gamble presented on the left or right.

The dependent measures were the proportion of choices of the 'experimental' over control gamble as a function of its probability of winning, size of the possible gains, and size of the possible losses ('proportionate choice'), and the mean deliberation time (ms) for these choices. We also measured participants' discrimination between differences in probability, gains and losses when choosing between gambles by calculating the absolute differences between the proportion of choices of the 'experimental' over control gamble when each of these factors was high (eg when the possible gains were large) and the proportion of choices when that factor was low (eg when the possible gains were small) (see Rogers et al, 2003 for more details).

As described previously (Rogers et al, 2003), we included two extra trial types that represented choices between gambles known to be subject to the non-normative biases of risk-aversion and risk-seeking behavior (see Kahneman and Tversky, 1979). The first such trial type was a 'gains-only' trial in which the participants were presented simultaneously with a guaranteed win of 40 points $v s$ a 0.5 chance of winning 80 points and a 0.5 chance of losing 0 points. Neither option involved any associated losses. By contrast, in the 'losses only' trial type, the participants were presented simultaneously with a guaranteed loss of 40 points $v s$ a 0.5 chance of losing 80 points and a 0.5 chance of losing 0 points. Neither option offered any associated gains. For both the 'gains-only' and 'losses-only' trials, the dependent measures were the proportion of trials on which the participants chose the guaranteed outcome and the associated mean deliberation time (ms) for these choices.

All 10 trial types were presented pseudorandomly within four blocks of trials. At the beginning of each block, participants were given 100 experimenter-defined points, and asked to make choices that would increase this amount by as much as possible. These points had no monetary value. Visual feedback was given after each choice and the revised points total was presented for $2 \mathrm{~s}$ before the next trial. Across the four blocks, there were eight repetitions of each 'experimental' gamble and eight repetitions of the 'gains-only' and 'losses-only' trial types.

\section{PROCEDURE}

Following recruitment, each participant arranged a suitable time to attend a testing session at a University Of Sussex laboratory. Upon arrival, each participant was provided with a consent form (including information on the experimental procedure) to read and sign. Participants were assigned an anonymous candidate number to ensure confidentiality. Each participant was recorded while undertaking the NART test. The participants completed the questionnaires ('Personal Details', then 'General Drug History'). Each participant then completed the MFF20, followed by the CARROT task. Participants were permitted to ask questions if they were unsure of the instructions that were included in the computer program. On completion of the CARROT task, participants were handed their total gains. Participants were then given printed instructions for the decision-making task, and once again permitted to ask questions if necessary. On completion of the test battery, each participant was given a short debriefing as to the purpose of the experiment. Advice regarding drug help groups was provided on the debriefing form.

\section{Data Analysis}

All the data were analyzed with SPSS (Version 11.0; SPSS Inc., Cary, NC). The measures of the decision-making task were the proportions of trials on which participants chose the 'experimental' over the control gamble ('proportionate choice'), and the deliberation time (ms) associated with these choices. The proportionate choice data were arcsinetransformed, as is appropriate whenever the variance of a measure is proportional to its mean (Howell, 1992); however, all of the data reported in the text, figures, and tables describe untransformed values. The results were analyzed using multifactorial repeated measures ANOVAs with the between-subject factors of group (ecstasy, polydrug, and control), and the within-subject factors of probability of winning (high $v s$ low), size of possible gains (large $v s$ small), and size of possible losses (large $v s$ small). Discrimination measures for probability, gains, and losses were analyzed by ANOVAs with group as betweensubjects factors. The 'gains-only' and 'losses-only' trials were analyzed with group and gender as between-subject factors and trial type ('gains only' $v s$ 'losses only') as a within-subject factor. One-way ANOVA was used to compare group card sorting rates and the reward responsiveness index measures in the CARROT test and the three MMF20 measures (mean latency to first response; total number of errors committed; and the composite 'I' score). Estimates of effect size (partial eta-squared values) for each significant effect are reported. Post hoc group comparisons were conducted with the Tukey HSD test. Mean difference scores were derived for RDMT proportions of choices of the 'experimental' gamble over the control gamble when prospective odds, gains, or losses were high minus the proportions of choices of the 'experimental' gamble when prospective odds, gains, or losses were low. These measures were then correlated with the MFF20 index of impulsivity 'I' using Pearson's product moment correlation coefficient. Finally, the proportion of choices data were reanalyzed with the MFF20 'I' score treated as a covariate. Group differences 
in gender ratio were tested with the $\chi^{2}$ test, while group differences in education were compared with the KruskalWallis test.

\section{RESULTS}

\section{Age, Gender, Education, and IQ}

The three drug groups did not differ with respect to age, gender ratio, level of education achieved, or IQ (estimated from NART scores) (see Table 1).

\section{Drug Histories}

Analysis of the self-reported drug use histories of participants in the three groups indicated that they were not significantly different in terms of their duration of use of alcohol, and consumption of cigarettes or alcohol in the month prior to testing. The groups did not differ in the time elapsed since last use of alcohol or cigarettes. However, oneway ANOVA revealed a significant group difference for the duration of use of cigarettes $(\mathrm{F}(2,57)=7.553, p=0.001)$. Post hoc analysis with Tukey's HSD indicated that the 'ecstasy' group had smoked cigarettes for significantly longer than the 'Control' group $(p<0.001)$. Further analysis of self-reported drug use of participants in the two drugusing groups ('ecstasy' and 'polydrug' groups) showed that they did not differ in terms of the amount of cannabis or poppers (nitrite inhalants) consumed, or duration of cannabis use. Self-reported use of other classes of illicit drugs was too infrequent for statistical analysis (see Table 2). However, to control for the possible effects of other illicit drug use, subsequent analyses were repeated after excluding the eight Ecstasy users who also reported past use of cocaine. These eight participants included seven of the nine ecstasy users who also had used 'poppers', three of the six who had used amphetamine, three of the four who had used mushrooms, and all of the ecstasy users who reported past use of ketamine. This left three amphetamine users, two popper users and one mushroom user in the remaining ecstasy group-minimal frequencies of other drug use which are similar to the three popper users, one amphetamineuser and one mushroom user, in the polydrug group.

\section{Ecstasy Use}

The ecstasy group comprised 11 males and nine females. The mean $( \pm S D)$ : age of first use was 17.5 (1.28) years,

Table I Participant Characteristics

\begin{tabular}{lrrr}
\hline Mean (SD) & $\begin{array}{l}\text { Control } \\
(\mathbf{n}=19)\end{array}$ & $\begin{array}{c}\text { Polydrug } \\
(\mathbf{n}=\mathbf{2 0})\end{array}$ & $\begin{array}{c}\text { Ecstasy } \\
(\mathbf{n}=\mathbf{2 0})\end{array}$ \\
\hline Age & $21.68(1.65)$ & $22.57(1.64)$ & $21.88(0.7 \mathrm{I})$ \\
Gender ratio $^{\mathrm{a}}$ & $1.52(0.5 \mathrm{I})$ & $1.40(0.5 \mathrm{I})$ & $1.45(0.5 \mathrm{I})$ \\
Education $^{\mathrm{b}}$ & $2.52(0.62)$ & $2.55(0.76)$ & $2.15(0.93)$ \\
${\text { Est. } \mathrm{QQ}^{\mathrm{c}}}$ & $111.8(5.66)$ & $112.1(5.35)$ & $111.2(6.05)$ \\
\hline
\end{tabular}

aMales coded as 1; females as 2 .

${ }^{\mathrm{b}} \mathrm{I}=$ GCSE, 2 =A-level, $3=$ Degree + .

'Estimated from NART scores. duration of use was 4.04 (1.30) years, and time since last use was 23.48 (16.57) days. Seven of these participants reported taking ecstasy within the week before testing. However, none had taken it for at least 5 days prior to testing. Ecstasy consumption ranged from a 60 to 288 tablets. Mean $( \pm S D)$ : dose per session was $2.38(0.76)$, frequency of use per month was $1.43(0.75)$ tablets, and estimated lifetime ecstasy consumption was $151.20( \pm 67.15)$ tablets.

\section{Matching Familiar Figures Test (MFF20)}

Analysis of the measures of MFF20 performance indicated that there were significant group differences in the mean latencies to first response $(F(2,56)=5.802$, $p=0.005$, partial eta squared $=0.172$ ), average total numbers of errors committed $[\mathrm{F}(2,56)=5.154, p=0.009$, partial eta squared $=0.155]$, and the derived 'I' scores $(F(2,56)=7.023$, $p=0.002$, partial eta squared $=0.201)$ (see Figure 2).

Latencies. Post hoc analysis with the Tukeys' HSD test indicated that 'ecstasy' participants delivered their first response significantly faster than 'control' participants $(p=0.004)$ '. However, there were no significant differences between the 'ecstasy' and 'polydrug' groups or between the 'control' and 'polydrug' groups (see Figure 2a).

Errors committed. Post hoc analysis with the Tukey's HSD test revealed that the 'ecstasy' group committed significantly more errors than both the 'control' group $(p=0.011)$ and the 'polydrug' group $(p=0.043)$. The 'control' and 'polydrug' groups did not differ significantly (see Figure $2 \mathrm{~b}$ ).

'I' score. Post hoc analysis with the Tukey's HSD test indicated that 'ecstasy' participants had significantly larger derived 'I' scores than both the 'control' group $(p=0.002)$ and the 'polydrug' group $(p=0.041)$. However the 'control' and 'polydrug' groups did not differ significantly (see Figure 2c).

After exclusion of the eight ecstasy users who used cocaine and other illicit drugs, the group differences in mean latencies to first response $(\mathrm{F}(2,48)=3.714, p=0.032$, partial eta squared $=0.134)$, total numbers of errors committed $(\mathrm{F}(2,48)=4.604, p=0.015$, partial eta squared $=$ $0.161)$, and 'I' scores (F $(2,48)=5.241, p=0.009$, partial eta squared $=0.179$ ), all remained significant. Post hoc analysis with the Tukeys' HSD test indicated that 'ecstasy' participants continued to deliver their first response significantly faster than 'control' participants $(p=0.025)$. However, there were no significant differences between the 'ecstasy' and 'polydrug' or between the 'control' and 'polydrug' groups. Post hoc analysis also revealed that the 'ecstasy' group continued to commit more errors than both the 'control' group $(p=0.015)$ and the 'polydrug' group $(p=0.045)$. The 'control' and 'polydrug' groups did not differ significantly. Finally, after exclusion of the eight ecstasy users who used cocaine Post hoc analysis indicated that the derived 'I' scores of 'ecstasy' participants continued to be significantly larger than those of the 'control' group ( $p=0.006)$, although those of the 'polydrug' 
Table 2 Means (SD) for Self-Reported Drug Consumption

'Control' group $(\mathrm{N}=19)$

'Polydrug' group $(N=20)$

'Ecstasy' group $(\mathbf{N}=\mathbf{2 0})$

Alcohol (units)

Participants reporting use

Consumed per month

Duration of use (years)

\section{Cigarettes}

Participants reporting use

Smoked per week

19

Duration of use (years)

Cannabis (joints)

Participants reporting use

Smoked per month

Duration of use (years)

Poppers (hits)

Participants reporting use

Consumed per year

Duration of use (years)

Amphetamine (g)

Participants reporting use

Consumed per year

Duration of use (years)

Cocaine (g)

Participants reporting use

Consumed per year

Duration of use (years)

LSD (trips)

Mushrooms (hits)

Participants reporting use

Consumed per year

Duration of use (years)

Ketamine (g)

Participants reporting use

Consumed per year

Duration of use (years)

19

0

0

0

0
$88.49(37.03)$

$6.95(2.35)$

$66.07(50.52)$

$4.23(3.13)$

20

$91.43(48.78)$

$6.39(2.98)$

0

3

$1.33(0.58)$

$0.39(0.53)$

0

0.50

0.08

0

$5.50(3.47)$

$2.19(1.39)$

1

0.5

0.08

0

20

$113.97(81.76)$

$8.05(1.50)$

20

$63.65(37.38)$

$8.18(2.11) *$

20

$79.55(80.13)$

$7.70(2.04)$

9

$3.56(2.13)$

$1.04(1.05)^{*}$

6

$1.53(0.76)$

$2.21(2.26)$

0

$7.00(5.57)$

$2.86(3.65)$ 3

*Significantly different at $p=0.05$.

group and 'control' and 'polydrug' groups did not differ significantly.

\section{CARROT}

There were no group differences in reward responsiveness in the CARROT test $(\mathrm{F}(2,58)=2.47, \mathrm{NS})$.

\section{Risky Decision-Making}

Proportionate choice. For technical reasons, the data from one participant in the control group was recorded inaccurately. Therefore, we excluded this participant's data from the analyses. All participants chose the 'experimental' gamble significantly more often when its probability of winning was high compared to when it was low 


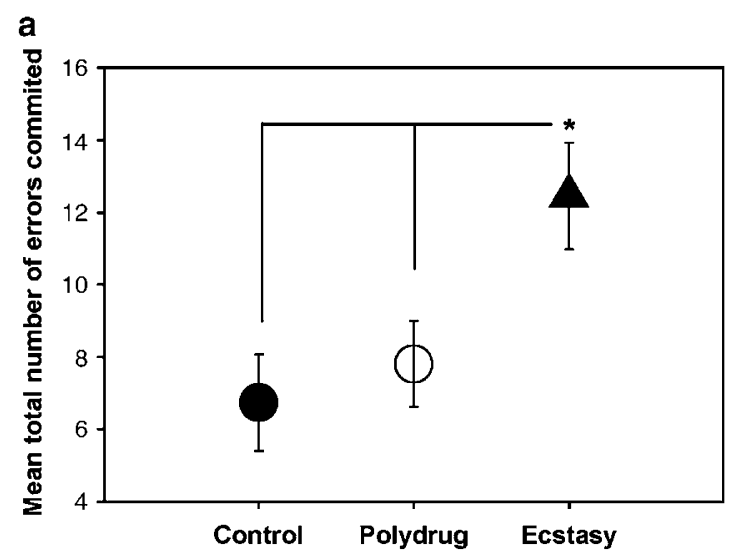

b
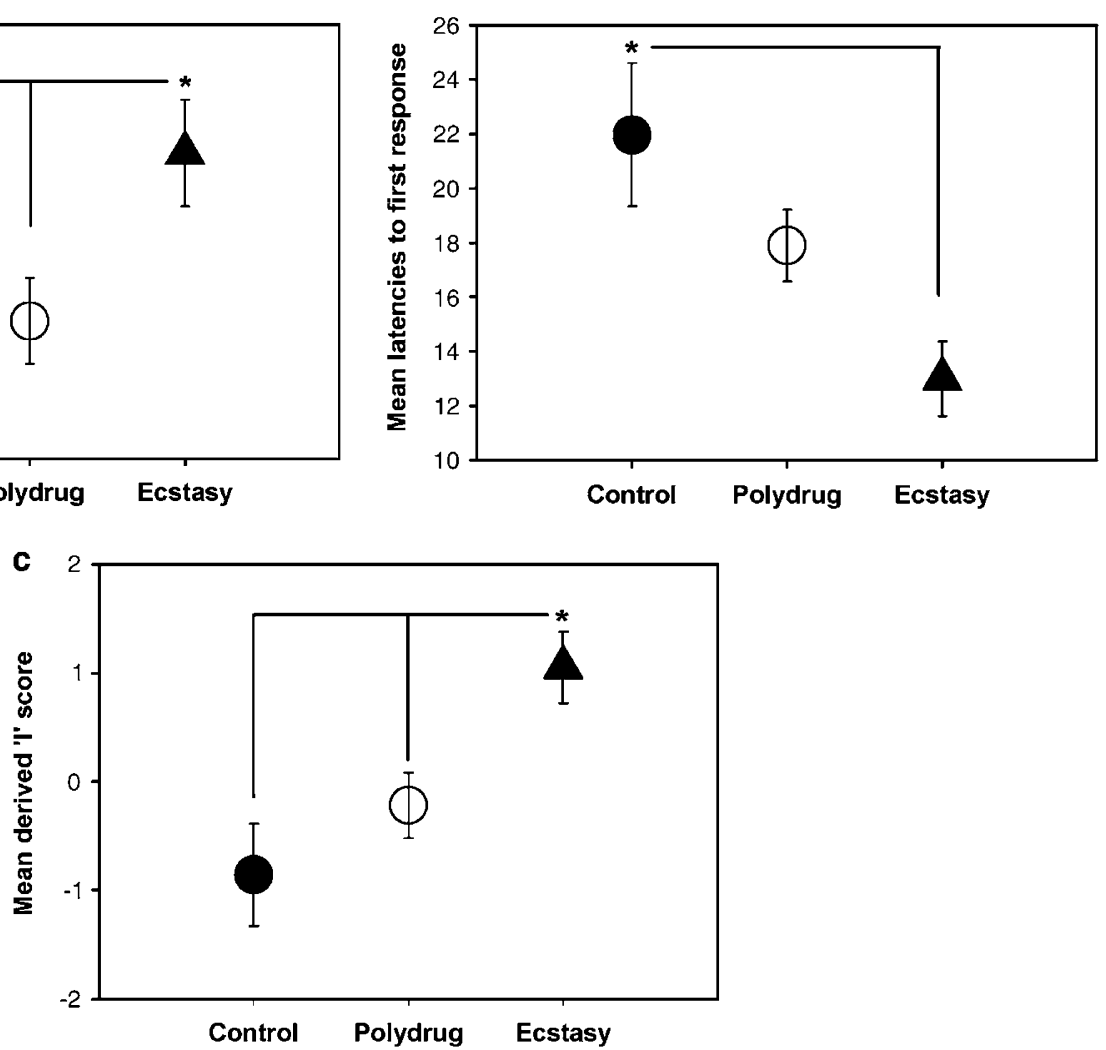

Figure 2 Group differences in impulsivity scores for the three experimental scores. (a) Mean total number of errors committed in the Matching Familiar Figures test. The ANOVA indicated that there were significant group differences in mean total numbers of errors committed $(F(2,56)=5.154, p=0.009)$. Tukey's HSD post hoc analysis revealed that the 'ecstasy' group committed significantly more errors than both the 'control' group ( $p=0.0 \mathrm{I} I)$ and the 'polydrug' group $(p=0.043)$. (b) Mean latencies to first response. The ANOVA indicated that there were significant group differences in the mean latencies to first response $(F(2,56)=5.802, p=0.005)$. Tukey's HSD post hoc analysis revealed that 'ecstasy' participants delivered their first response significantly faster than 'control' participants $(p=0.004)$. (c) Mean derived 'l' scores. The ANOVA indicated that there were significant group differences in mean derived 'I' scores ( $F(2,56)=7.023, p=0.002)$. Tukey's HSD post hoc analysis revealed that 'ecstasy' participants had significantly larger derived 'I' scores than both the 'control' group $(p=0.002)$ and the 'polydrug' group $(p=0.041)$.

$(\mathrm{F}(1,56)=440.479, p<0.001)$ (see Figure 3a), and when the possible gains were large compared to when they were small $(\mathrm{F}(1,56)=127.434, p<0.001)$ (see Figure $3 \mathrm{~b}$ ). Furthermore, all participants chose the 'experimental' gamble significantly less often when possible losses were large compared to when they were small (F $(1,56)=159.877, p<0.001)$ (see Figure $3 \mathrm{c}$ ). However, this discrimination between large and small prospective gains, and between large and small prospective losses, when choosing between gambles was markedly reduced in the ecstasy users compared to the polydrug users and controls as indicated by significant two-way interactions between group and size of possible gains $(\mathrm{F}(2,56)$ $=8.116, p=0.001$, partial eta squared $=0.225)$ and between group and the size of possible losses $(F(2,56)=5.163$, $p=0.001$, partial eta squared $=0.156)$ respectively.

When the eight Ecstasy users who reported past use of cocaine and other illicit drugs were excluded from the analyses, the reduced discrimination between large and small potential gains in the ecstasy users compared to the polydrug and controls remained significant $(F(2,48)=$ $3.577, p=0.036$, partial eta squared $=0.130$ ); however, the reduced discrimination between large and small potential losses was now nonsignificant $(\mathrm{F}(2,48)=1.584$, NS, partial eta squared $=0.062$ ).
Mean difference scores between the proportions of choices of the 'experimental' gamble over the control gamble when prospective gains were large minus the proportions of choices of the 'experimental' gamble when prospective gains were small were negatively correlated with MFF20 'I' scores $(r=-0.380, p<0.003)$. Mean difference scores between the proportions of choices of the 'experimental' gamble over the control gamble when prospective losses were small minus the proportions of choices of the 'experimental' gamble when prospective losses were large were also negatively correlated with MFF20 'I' scores $(r=-0.294, p<0.024)$ (see Figure 4$)$

When the MFF20 index of impulsivity ' $\mathrm{I}$ ' was treated as a covariate on the complete data set, the two-way interaction between group and size of possible gains remained significant $(\mathrm{F}(2,55)=5.478, p=0.007$, partial eta squared $=$ 0.166 ), while the two-way interaction between group by size of possible losses just failed to reach statistical significance $(\mathrm{F}(2,55)=2.955, p=0.060$, partial eta squared $=0.097)$.

Deliberation times. There were no significant between group differences for the deliberation times in this task. Overall the mean latencies in the MFF20 task correlated 
a

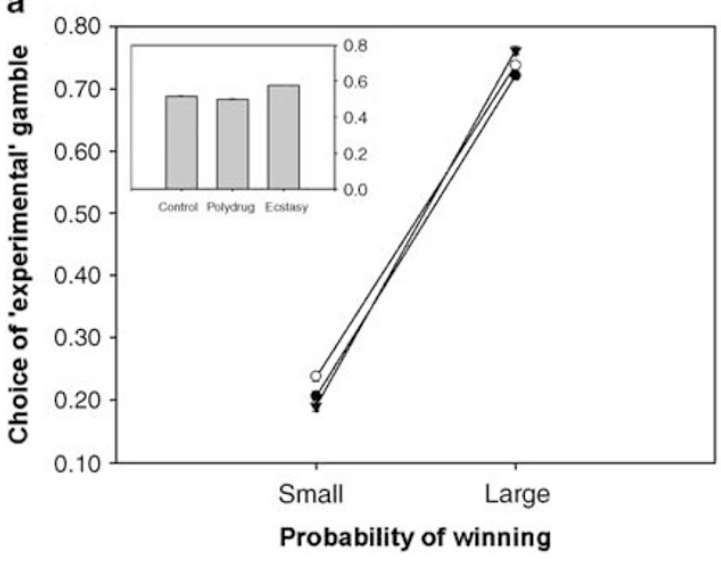

b

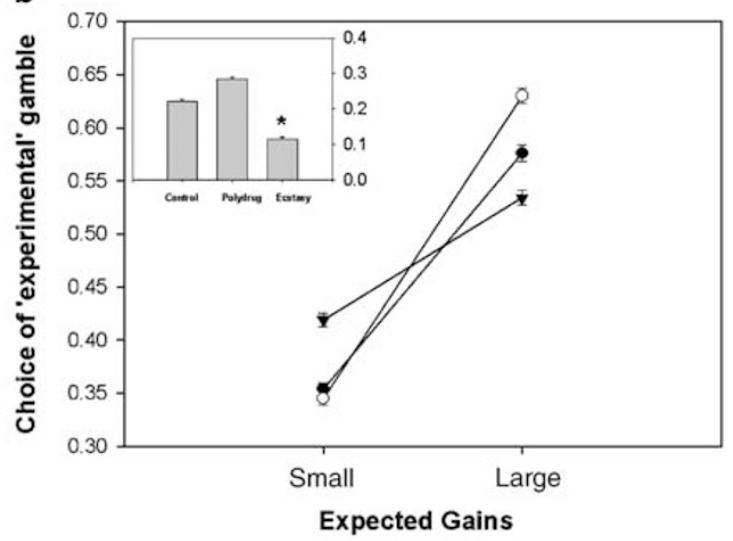

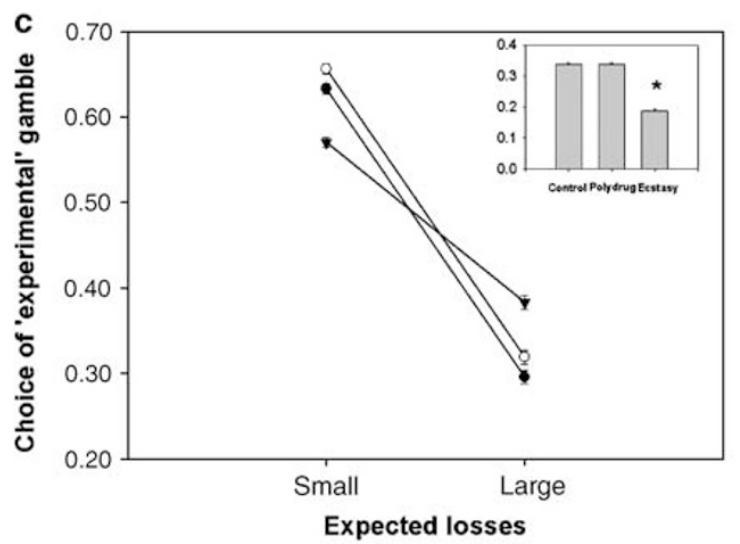

Figure 3 Proportion of choices of the 'experimental' over the control gamble by the three experimental groups (controls, polydrug, ecstasy) for the factors. (a) High vs low probability of winning (inset: the mean difference between the proportions of choices of the 'experimental' over the control gamble when its probability of winning was high minus the proportions of choices of the 'experimental' gamble when its probability of winning was low); (b) high vs low prospective gains. Group $\times$ prospective gains (high vs low): $(F(I, 56)=127.43, p<0.00 I)$ (inset: the mean difference between the proportions of choices of the 'experimental' gamble over the control gamble when prospective gains were high minus the proportions of choices of the 'experimental' gamble when prospective gains were low: $(F(2,58)=8.116, p=0.00 \mathrm{I})$ ); (c) high vs low prospective losses. Group $\times$ prospective losses (high vs low): $(F$ $(I, 56)=159.88, p<0.00 I)$ (inset: the mean difference between the proportions of choices of the 'experimental' over the control gamble when prospective losses were high minus the proportions of choices when prospective losses were low: $(F(2,58)=5.16, p=0.009))$.

positively with the mean latencies in the risky decisionmaking task $(r=0.284, p<0.05)$.

\section{'Gains-Only' vs 'Losses-Only' Trials}

Proportionate choice. Participants chose the guaranteed outcome significantly more often on the 'gains-only' trials (offering a choice between a certain gain on 40 points and a 0.50 chance of 80 or 0 points) than on the losses-only trials (offering a certain loss of 40 points or a 0.5 chance of a loss of 80 or 0 points) $(\mathrm{F}(1,56)=130.631, p<0.001)$. There were no significant main effects of group or group by trial-type interactions in risk aversion when choosing between gains and risk seeking when choosing between losses. In the losses-only condition the mean (SD) proportion choosing the guaranteed outcome for the three groups were: ecstasy $0.2250( \pm 0.2420)$, polydrug 0.1438 $( \pm 0.2443)$, and control $0.2105( \pm 0.1912)$. In the gains-only condition, the mean (SD) proportion choosing the guaranteed outcome for the three groups were: ecstasy 0.7688 $( \pm 0.2729)$, polydrug $0.8000( \pm 0.2850)$, and control 0.8224 $( \pm 0.2613)$.
Deliberation times. All participants made significantly slower decisions on the 'gains only' trials compared to the 'losses-only' trials $(\mathrm{F}(1,56)=26.54, p<0.001)$, but there was no significant main effect of group and no group by trial-type interaction. In the losses-only condition, the mean (SD) deliberation time in $\mathrm{ms}$ for the three groups were: ecstasy $2410( \pm 1373)$, polydrug $2392( \pm 868)$, and control $3044( \pm 3717)$. In the gains-only condition, the mean (SD) deliberation time in $\mathrm{ms}$ for the three groups were: ecstasy $3589( \pm 1948)$, polydrug $4266( \pm 2063)$, and control 4528 $( \pm 2120)$.

\section{DISCUSSION}

The present results demonstrate a pattern of elevated impulsivity and altered risky choice in recreational ecstasy users compared to polydrug users (who had similar cannabis histories but had never taken ecstasy) and nondrug using control participants (who had never taken illicit drugs). These effects are not attributable to demographic or psychometric factors as the three subject groups 

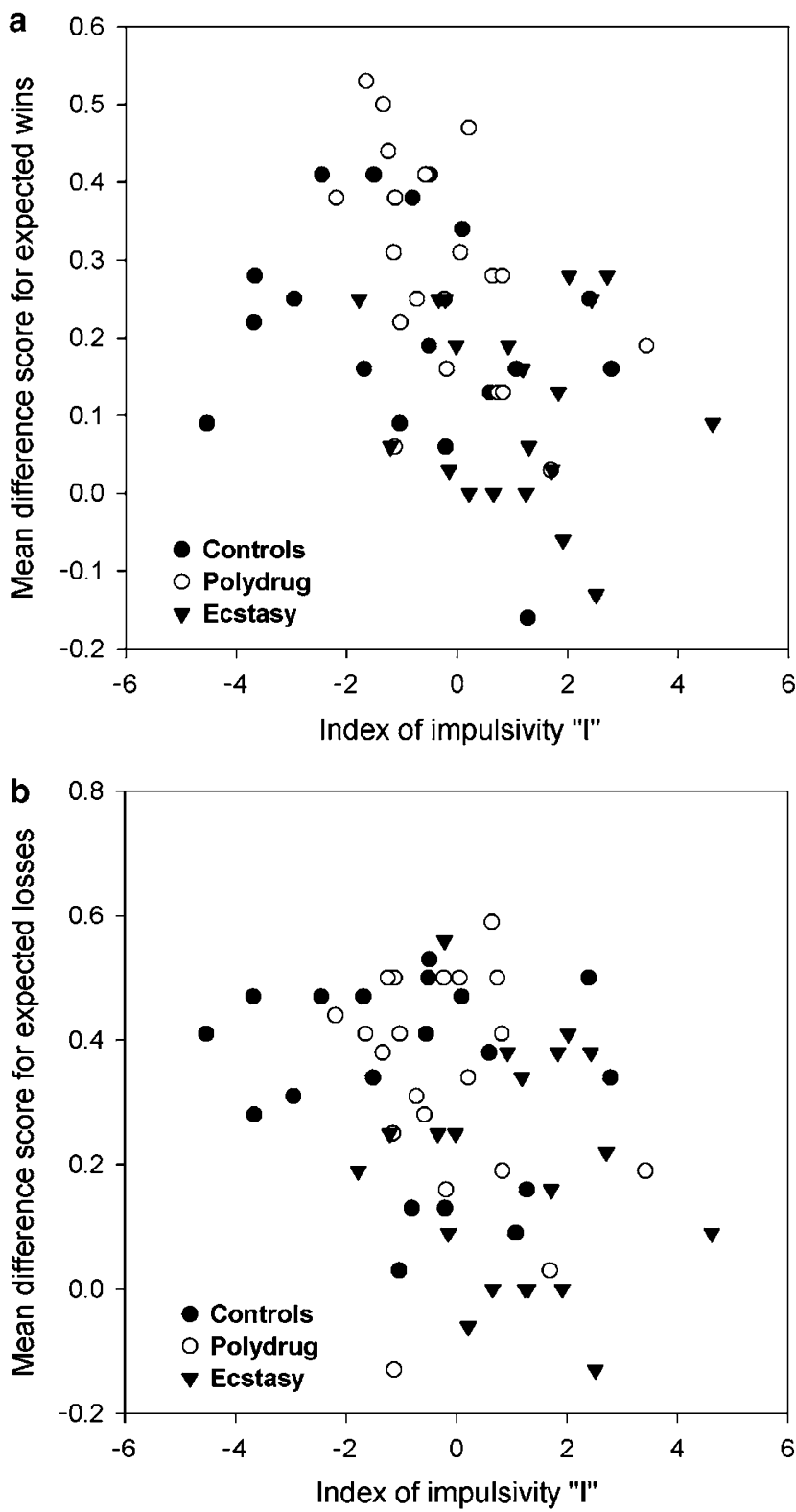

Figure 4 Relation between the proportion of choices of the 'experimental' over the control gamble and MFF20 impulsivity index 'I' scores. The proportion of times participants chose the 'experimental' gamble over the control gamble in the decision-making task was recorded. The proportion of times when prospective gains, or losses were low, was then subtracted from the proportions when prospective wins, or losses, were high to obtain mean difference scores for gains and losses. (a) The derived mean difference scores for prospective gains were negatively correlated with MFF20 impulsivity index 'I' scores $(r=-0.380, p<0.003)$. (b) The derived mean difference scores for prospective losses were also negatively correlated with MFF20 impulsivity index 'I' scores $(r=-0.294, p<0.024)$

were closely matched for age, gender ratio, education, and estimated premorbid IQ. They were not attributable to past use of cocaine or ketamine, as the primary findings generally remained significant when all the ecstasy users who also used cocaine and ketamine were excluded. Furthermore, they were unlikely to result from confounding by the use of other illicit drugs because, after the exclusion of the latter participants, the residual incidence of past use of other illicit drugs was similar between the two drug using groups. Neither is it likely that these results reflect a loss of motivation for financial incentive in the ecstasy users since there were no significant group differences in CARROT (Powell et al, 1996) performance.

The ecstasy users committed significantly more errors, on the Matching Familiar Figures test (MFF20; Cairns and Cammock, 1978), compared to the polydrug users and drug-naïve participants, and delivered their first response significantly faster than the drug-naïve participants. The responses of all participants, and controls in particular, tended to be delayed compared to participants in our previous studies (Morgan, 1998; Morgan et al, 2002), although the error rate was similar across all studies. This may be attributable to the fact that the novel computerized version of the MFF20 task employed in the present study requires a keyboard response rather than a simple pointing response. Nevertheless, the composite 'I' scores-derived by subtracting the standard score of the mean latency to first response from the standard score of the total number of errors committed $\left(Z_{\mathrm{e}}-Z_{\mathrm{l}}\right)$ - were also significantly increased in the ecstasy users compared to participants in the other two groups. Thus, the present findings are generally in agreement with our earlier published findings (Morgan, 1998; Morgan et al, 2002). They are also consistent with Butler and Montgomery (2004), who reported that heavy ecstasy users scored higher on a risk-taking measure than non-drug users, cannabis users, and low ecstasy users. However, the present results are at variance with those of Gouzoulis-Mayfrank et al (2003), who reported that there was no difference in Go/NoGo performance between nonusers, moderate, and heavy ecstasy users despite evidence that the ecstasy users exhibited poorer memory and working memory performance. This discrepancy may arise because the type of deficient inhibition modelled by the Go/NoGo task is qualitatively distinct, and has dissociable neural and neuropharmacological substrates from the 'reflection-impulsivity' subtype of impulsivity modelled by the MFF20 (Evenden, 1999; Winstanley et al, 2004).

Effective decisions involving uncertain benefits and penalties require decision-makers to integrate different reinforcement cues including the size of possible gains (rewards), the size of possible losses (punishments), and the probability of these outcomes. Therefore, examining how samples of drug users use such information while selecting between actions associated with motivationally significant outcomes may reveal how altered processing of reinforcement signals is associated with failing behavioral regulation in substance misuse. In the risky choice task used in the present study (Rogers et al, 2003), the 'ecstasy' users exhibited reduced discrimination between magnitudes of both possible gains and losses associated with different choices compared to the nondrug using 'control' participants, and 'polydrug' participants. This suggests that the ecstasy users allocated less attentional processing of potential rewards and punishments while choosing between actions associated with uncertain outcomes.

There is already preliminary evidence to suggest that regular ecstasy use is associated with impairments in attention. For example, Gouzoulis-Mayfrank et al (2000) reported that although ecstasy users were unimpaired in 
simple tests of attention (alertness), they performed worse than cannabis users and nonusers in the more complex tests of attention. Fox et al (2002) reported that ecstasy users were significantly impaired on a recognition task for complex visual patterns and spatial working memory, as a function of task difficulty rather than systematic search strategy. Zakzanis et al (2002) reported differences in performance between ecstasy users and controls in a subtest of the 'Test of Everyday Attention' (the map search task) and that the number of ecstasy tablets used was negatively correlated with performance on a number of attention tasks. Jacobsen et al (2004) reported that adolescent ecstasy users had significantly prolonged reaction times during tests of selective and divided attention, and failed to deactivate the left hippocampus normally during high verbal working memory load compared to matched ecstasy-naïve controls. Finally, Mejias et al (2005) reported that ecstasy users used longer latencies than controls to detect infrequent stimuli and did not exhibit a shorter latency N200 component (which is involved in attention orienting associated with the detection of stimulus novelty) in response to such stimuli. This absence of delay was interpreted as evidence of attentional deficits in ecstasy users.

Further research is needed to elucidate the possible importance of altered attention towards reinforcement cues in risky choice within the wider neuropsychological profile of current ecstasy users. However, we note the statistical association between discrimination between gains and losses on the one hand, and high reflection impulsivity as measured by the MFFT20 on the other hand, in the ecstasy users, is consistent with the possibility that their altered decisionmaking is one manifestation of a tendency to respond without fully sampling all of the information relevant to the choice. This finding is substantiated by a recent study of the risky decision-making in current ecstasy users, ex-users users, polydrug controls, and drug-naive controls using a relatively less sensitive version of the task used in the present study in which the probability of good outcomes varied between 0.8 and 0.2 rather than 0.66 and 0.33 (Roiser et al, 2005). The samples were carefully matched for polydrug use and indicated only modest evidence of neuropsychological deficits in those participants who were currently using ecstasy. However, within this group but not the control groups, self-reported impulsivity was negatively associated with reduced attention towards possible gains when deciding between risky gambles. Therefore, these data suggest that trait impulsivity may mediate some of the cognitive dysfunctions reported in ecstasy users (Morgan, 2000). If the specific connection between heightened impulsivity and altered attention to reinforcement can be substantiated, the pattern of choices observed here in ecstasy users might be remediated by manipulations that delay users' choices or that force ecstasy users to process reinforcement information to a greater extent.

Accumulating evidence suggests that emotional decisionmaking depends upon an interconnected circuitry involving the orbitofrontal and medial PFC (including the rostrocingulate cortex), amygdala, and striatum (Bechara et al, 1996; Rogers et al, 2004b), as well as its modulation by monoaminergic systems. Manipulation of the serotonin, noradrenaline, and dopamine has been found to alter the processing of reinforcement cues, while healthy participants perform the risky choice used in the current study (Rogers et al, 2003, 2004a; Scarna et al, 2005). Of particular relevance is the finding that dietary depletion of Ltryptophan was associated with reduced attention to possible gains but not losses (Rogers et al, 2003), suggesting that serotonin mediates appetitive aspects of risky choice. Consistent with previous claims of serotonergic deficits in ecstasy users (eg McCann et al, 1998; Semple et al, 1999; Reneman et al, 2000), the present study demonstrated reduced attention to gains while such participants made risky choices. The present finding that the ecstasy users also showed reduced attention to possible losses, as well as gains, suggest that serotonin dysfunction might also undermine the processing of aversive as well as appetitive cues in risky choice. On the other hand, it may result from confounding by the additional effects of other illicit drugs on other neurotransmitter systems. When the eight Ecstasy users who used cocaine and other illicit drugs were excluded from the analyses, discrimination between potential gains continued to be significantly reduced in the remaining 'purer' ecstasy users, whereas discrimination between losses was no longer significant. This is more consistent with the view that serotonin depletion is associated with reduced attention to possible gains but not losses (Rogers et al, 2003) in the risky choice task used in the current study.

There were some methodological limitations with the present study that characterize research in this area. These included problems with sampling and reliability of selfreported drug histories (eg Morgan, 2000; Wolff et al, 1995). The present study did not employ assays to confirm selfreported alcohol and substance use prior to testing. However, Thomasius et al (2003) reported good concordance between self-reported ecstasy use and the results of hair analyses in their study, suggesting that the present drug histories are also likely to be reasonably reliable. Nevertheless, it would be desirable to employ hair sample assays to corroborate self-reported drug histories in future studies of this type. It would also be interesting to investigate the role of age and gender by testing larger samples of adolescents, as well as young adult participants, who indicate that ecstasy is their drug of choice and who only co-use cannabis.

Finally, there is some evidence that serotonergic deficits in 'ecstasy' users can recover after prolonged abstinence (Reneman et al, 2001; Buchert et al, 2004; Thomasius et al, 2003) and mixed evidence for the persistence of neuropsychological deficits in ex-ecstasy users (Morgan et al, 2002). Therefore, further research will be needed to examine whether the comparable patterns of risky choice can be observed in drug users with a history of ecstasy use but no current use, and to explore whether such effects depend upon individual differences in traits such as impulsiveness that predispose individuals to altered emotional choice and possibly substance misuse (Robbins and Everitt, 1996).

\section{ACKNOWLEDGEMENTS}

We would like to acknowledge the generous contribution of Paris Val-Baker in programming the computerized version of the MFF20. 


\section{REFERENCES}

Bechara A, Dolan S, Denburg N, Hindes A, Anderson SW, Nathan PE (2001). Decision-making deficits, linked to a dysfunctional ventromedial prefrontal cortex, revealed in alcohol and stimulant abusers. Neuropsychologia 39: 376-389.

Bechara A, Tranel D, Damasio H, Damasio AR (1996). Failure to respond autonomically to anticipated future outcomes following damage to prefrontal cortex. Cereb Cortex 6: 215-225.

Buchert R, Thomasius R, Wilke F, Petersen K, Nebeling B, Obrocki $\mathrm{J}$ et al (2004). A voxel-based PET investigation of the long-term effects of 'ecstasy' consumption on brain serotonin transporters. Am J Psychiatry 161: 1181-1189.

Butler GK, Montgomery AM (2004). Impulsivity, risk taking and recreational 'ecstasy' (MDMA) use. Drug Alcohol Depend 76: $55-62$.

Cairns E, Cammock T (1978). Development of a more reliable version of the Matching Familiar Figures Test. Dev Psychol 13: 555-560.

Coccaro EF (1989). Central serotonin and impulsive aggression. Br J Psychiatry 155: 52-62.

Evenden JL (1999). Varieties of impulsivity. Psychopharmacology 146: $348-361$.

Fishbein DH, Lozovsky D, Jaffe JH (1989). Impulsivity, aggression, and neuroendocrine responses to serotonergic stimulation in substance abusers. Biol Psychiatry 25: 1049-1066.

Fox HC, McLean A, Turner JJD, Parrott AC, Rogers R, Sahakian BJ (2002). Neuropsychological evidence of a relatively selective profile of temporal dysfunction in drug-free MDMA ('ecstasy') polydrug users. Psychopharmacology 162: 203-214.

Gouzoulis-Mayfrank E, Daumann J, Tuchtenhagen F, Pelz S, Becker S, Kunert H-K et al (2000). Impaired cognitive performance in drug free users of recreational ecstasy (MDMA). J Neurol Neurosurg Psychiatry 68: 719-725.

Gouzoulis-Mayfrank E, Thimm B, Rezk M, Hensen G, Daumann J (2003). Memory impairment suggests hippocampal dysfunction in abstinent ecstasy users. Progr Neuropsychopharmacol Biol Psychiat 27: 819-827.

Grant S, Contoreggi C, London ED (2000). Drug abusers show impaired performance in alaboratory test of decision making. Neuropsychologia 38: 1180-1187.

Green AR, Cross AJ, Goodwin GM (1995). Review of the pharmacology and clinical pharmacology of 3,4-methylenedioxymethamphetamine (MDMA or ecstasy). Psychopharmacology 119: $247-260$.

Hanson KL, Luciana M. (2004). Neurocognitive function in users of MDMA: the importance of clinically significant patterns of use. Psychol Med 34: 229-246.

Harris GC, Aston-Jones G (2003). Altered motivation and learning following opiate withdrawal: evidence for prolonged dysregulation of reward processing. Neuropsychopharmacology 28: 865-871.

Howell DC (1992). Statistical Methods for Psychology, 3rd edn. Wadsworth: Belmont, CA.

Jacobsen LK, Mencl WE, Pugh KR, Skudlarski P, Krystal JH (2004). Preliminary evidence of hippocampal dysfunction in adolescent MDMA ('ecstasy') users: possible relationship to neurotoxic effects. Psychopharmacology 173: 383-390.

Johnston LD, O’Malley PM, Bachman JG (2000). The Monitoring the Future National Survey Results on Adolescent Drug Use: Overview of the Key Findings, 1999 (NIH Publication No. 00-4690). National Institute on Drug Abuse: Rockville, $\mathrm{MD}, 56 \mathrm{pp}$.

Kagan J, Rosman BL, Day D, Albert J, Phillips W (1964). Information processing in the child: significance of analytic and reflective attitudes. Psychol Mono 78: 1-37.

Kahneman D, Tversky A (1979). 'Prospect theory: an analysis of decision under risk'. Econometrica 47: 263-291.
Kish SJ, Furukawa Y, Ang L, Vorce SP, Kalasinsky KS (2000). Striatal serotonin is depleted in brain of a human MDMA (ecstasy) user. Neurology 55: 294-296.

Linnoila M, Virkkunen M, George T, Higley D (1993). Impulse control disorders. Int Clin Psychopharmacol 8: 53-56.

McCann UD, Mertl M, Eligulashvili V, Ricaurte GA (1999). Cognitive performance in ( \pm ) 3,4-methylenedioxymethamphetamine (MDMA; 'ecstasy') users: a controlled study. Psychopharmacology 143: 417-425.

McCann UD, Ridenour A, Shaman Y, Ricuarte GA (1994). Serotonin neurotoxicity after MDMA ('ecstasy'): a controlled study in humans. Neuropsychopharmacology 10: 129-138.

McCann UD, Szabo Z, Scheffel U, Dannals RF, Ricaurte GA (1998). Positron emission tomographic evidence of toxic effect of MDMA ('ecstasy') on brain serotonin neurons in human beings. Lancet 352: 1433-1437.

Mejias S, Rossignol M, Debatisse D, Streel E, Servais L, Guerit JM et al (2005). Event-related potentials (ERPs) in ecstasy (MDMA) users during a visual oddball task. Biol Psychol 69: 333-352.

Messer SB, Brodzinsky DM (1981). Three-year stability of reflection-impulsivity in young adolescents. Dev Psychol 17: 848-850.

Morgan MJ (1998). Recreational use of ecstasy (MDMA) is associated with elevated impulsivity. Neuropsychopharmacology 19: $252-264$.

Morgan MJ (1999). Memory deficits associated with recreational use of 'ecstasy' (MDMA). Psychopharmacology 141: 30-36.

Morgan MJ (2000). Ecstasy (MDMA): a review of its possible persistent psychological effects. Psychopharmacology 152: 230-248.

Morgan MJ, McFie L, Fleetwood LH, Robinson JA (2002). 'Ecstasy' (MDMA): are the psychological problems associated with its use reversed by prolonged abstinence? Psychopharmacology 159: 294-303.

Nelson HE (1982). National Adult Reading Test. Nelson Publishing Company Ltd: Windsor, England.

Powell J, Al-Adawi S, Morgan J, Greenwood R (1996). Motivational deficits after brain injury: effects of bromocriptine in 11 patients. J Neurol Neurosurg Psychiatry 60: 416-421.

Reneman L, Booij J, de Bruin K, Reitsma JB, de Wolff FA, Gunning WB et al (2001). Effects of dose, sex, and long-term abstention from use on toxic effects of MDMA (ecstasy) on brain serotonin neurons. Lancet 358: 1864-1869.

Reneman L, Booij J, Schmand B, Brink W, Gunning B (2000). Memory disturbances in 'ecstasy' users are correlated with an altered serotonin neurotransmission. Psychopharmacology 148: 322-324.

Ricaurte GA, Katz JL, Martello MB (1992). Lasting effects of $( \pm)$ 3,4-methylenedioxymeth-amphetamine on central serotonergic neurons in non-human primates. J Pharmacol Exp Ther 261: 616-622.

Ricaurte GA, Yuan J, McCann UD (2000). 3,4-Methylenedioxymethamphetamine ('ecstasy')-induced neurotoxicity: studies in animals. Neuropsychobiology 42: 5-10.

Robbins TW, Everitt BJ (1996). Neurobehavioural mechanisms of reward and motivation. Curr Opin Neurobiol 6: 228-236.

Rodgers J (2000). Cognitive performance amongst recreational users of 'ecstasy'. Psychopharmacology 151: 19-24.

Rogers RD, Blackshaw AJ, Middleton HC, Matthews K, Hawttin K, Crowley C et al (1999b). Tryptophan depletion impairs stimulusreward learning while methylphenidate disrupts attentional control in healthy young adults: implications for the monoaminergic basis of impulsive behaviour. Psychopharmacology 146: 482-491.

Rogers RD, Everitt BJ, Baldacchino A, Blackshaw AJ, Swainson R, Bakera NB et al (1999a). Dissociable deficits in the decisionmaking cognition of chronic amphetamine abusers, opiate abusers, patients with focal damage to prefrontal cortex, and 
tryptophan-depleted normal volunteers: evidence for monoaminergic mechanisms. Neuropsychopharmacology 20: 322-339.

Rogers RD, Lancaster M, Wakeley J, Bhagwagar Z. (2004a). Effects of beta-adrenoceptor blockade on components of human decision-making. Psychopharmacology 172: 157-164.

Rogers RD, Ramnani N, MacKay C, Wilson J, Jezzard P, Carter CS et al (2004b). Distinct portions of anterior cingulate cortex and medial prefrontal cortex are activated by reward processing in separable phases of decision-making cognition. Biol Psychiatry 55: 594-602.

Rogers RD, Tunbridge EM, Bhagwagar Z, Drevets WC, Sahakian BJ, Carter CS (2003). Tryptophan depletion alters decisionmaking of healthy volunteers through altered processing of reward cues. Neuropsychopharmacology 28: 153-162.

Roiser JP, Rogers RD, Sahakian BJ (2005). Neuropsychological function in ecstasy users: a study controlling for poly-drug use. Psychopharmacology, 15 September [Epub ahead of print].

Salkind NJ, Wright J (1977). The development of reflectionimpulsivity and cognitive efficiency: an integrated model. Hum Dev 20: 377-387.

Scarna A, McTavish SFB, Cowen PJ, Goodwin GM, Rogers RD (2005). The effects of a branched chain amino acid mixture supplemented with tryptophan on biochemical indices of neurotransmitter function and decision-making. Psychopharmacology 179: 761-768.

Semple DM, Ebmeier KP, Glabus MF, O'Carroll RE, Johnstone EC (1999). Reduced in vivo binding to the serotonin transporter in the cerebral cortex of MDMA ('ecstasy') users. Br J Psychiatry 175: 63-69.

Soubrié P (1986). Serotonergic neurons and behavior. J Pharmacol 17: $107-112$.

Steele TD, McCann UD, Ricuarte GA (1994). 3,4-Methylenedioxymeth-amphetamine (MDMA, 'ecstasy'): pharmacology and toxicology in animals and humans. Addiction 89: 539-551.
Tasker T, Raw M, McNeill A (1999). Drug Realities: A Summary of the Results of the 1996 National Drugs Campaign Survey. Health Education Authority: London.

Thomasius R, Petersen K, Buchert R, Andresen B, Zapletalova P, Wartberg L et al (2003). Mood, cognition and serotonin transporter availability in current and former ecstasy (MDMA) users. Psychopharmacology 167: 85-96.

Verkes RJ, Gijsman HJ, Pieters MS, Schoemaker RC, de Visser S, Kuijpers $M$ et al (2001). Cognitive performance and serotonergic function in users of ecstasy. Psychopharmacology 153: 196-202.

von Geusau N, Stalenhoef P, Huizinga M, Snel J, Ridderinkhof KR (2004). Impaired executive function in male MDMA ('ecstasy') users. Psychopharmacology 175: 331-341.

Wareing M, Fisk JE, Murphy PN (2000). Working memory deficits in current and previous users of MDMA ('ecstasy'). Br J Psychol 91: 181-188.

Wareing M, Murphy PN, Fisk JE (2004). Visuospatial memory impairments in users of MDMA ('ecstasy'). Psychopharmacology 173: 391-397.

Wilson JM, Levey AI, Bergeron C, Kalasinsky K, Ang L, Peretti F et al (1996). Striatal dopamine, dopamine transporter, and vesicular monoamine transporter in chronic cocaine users. Ann Neurol 40: 428-439.

Winstanley CA, Dalley JW, Theobald DE, Robbins TW (2004). Fractionating impulsivity: contrasting effects of central 5-HT depletion on different measures of impulsive behavior. Neuropsychopharmacology 29: 1331-1343.

Wolff K, Hay AWM, Sherlock K, Conner M (1995). Contents of 'ecstasy'. Lancet 346: 1100-1101.

Zakzanis KK, Young DA, Radkhoshnoud NF (2002). Attentional processes in abstinent methylenedioxymethamphetamine (ecstasy) users. Appl Neuropsychol 9: 84-91. 